\title{
Kulturelle Differenz in der Gesundheitsversorgung
}

\author{
Michael Coors • Tim Peters • Ilhan Ilkilic
}

Online publiziert: 4. Juli 2018

(C) Springer-Verlag GmbH Deutschland, ein Teil von Springer Nature 2018

Die fächerübergreifende wissenschaftliche Auseinandersetzung mit dem Phänomen kultureller Differenz hat deutlich gemacht, dass kulturelle Identitäten - insbesondere als gruppenbezogene Identitäten - keine feststehenden, gewissermaßen natürlichen Größen sind. „Kulturen“ gibt es nicht ,an sich“, wie es ein essentialistischer Kulturbegriff nahelegt, sondern kulturelle Identitäten sind immer Ergebnis von komplexen Prozessen gesellschaftlicher Eigen- und Fremdzuschreibungen in zwischenmenschlichen Beziehungen (Wimmer 1996; Knipper 2014; Peters et al. 2014). Dennoch arbeiten wir im Alltag, gerade auch der medizinischen und pflegerischen Versorgung, immer auch mit gruppenbezogenen Zuschreibungen, wenn z. B. von türkischen oder von muslimischen Patientinnen oder Patienten oder von der ,polnischen Krankenschwester“ die Rede ist. Derartige Zuschreibungen sind nicht zwangsläufig problematisch, aber sie müssen in ihrer Vorläufigkeit als stereotype Zuschreibungen reflektiert werden. Sie können, mit den treffenden Worten von Michael Knipper (2014, S. 57), immer nur das ,,zeit- und situationsgebundene (Zwischen-)Ergebnis sozialer Beziehungen“" sein.

Problematisch werden diese Zuschreibungen gruppenbezogener kultureller Identitäten darum immer dann, wenn sie als feststehende Erklärungsmuster herhalten müssen, also den Charakter des Vorläufigen und Reversiblen verlieren. Dies gilt gerade auch in moralischen Konfliktsituationen. Dann wird aus ,dem muslimischen Patienten“ allzu leicht eine Chiffre für den ,schwierigen Patienten“, der sich nicht in-

PD Pastor Dr. theol. habil. M. Coors $(\bowtie)$

Zentrum für Gesundheitsethik (ZfG), Knochenhauerstr. 33, 30159 Hannover, Deutschland

E-Mail: michael.coors@evlka.de

Dr. phil. T. Peters

Zentrum für Medizinische Lehre, Ruhr-Universität Bochum, Bochum, Deutschland

Prof. Dr. (TR) Dr. phil. et med. habil. I. Ilkilic

Institut für Ethik und Geschichte der Medizin, Universitätsmedizin Istanbul, Istanbul, Türkei 
tegriere und die moralischen Standards „unserer Kultur“ nicht akzeptiere. Kulturelle Differenz, die in diesem Fall als religiöse Differenz konfiguriert ist, wird damit zu einer vermeintlich plausiblen Erklärung für einen moralischen Konflikt. Die Logik dahinter ist so einfach wie fatal: „Wir verstehen uns moralisch nicht, weil wir aus unterschiedlichen Kulturen kommen." Fatal ist daran, dass dieses Erklärungsmuster eines moralischen Konfliktes keinen Spielraum für eine gemeinsame Lösung lässt, außer durch eine Nivellierung der kulturellen Differenz auf Kosten der kulturellen Identität desjenigen, der in der Rolle ,des Fremden“ ist - also in diesem Beispiel des muslimischen Patienten. Damit verstärkt solch ein Erklärungsmuster interkultureller moralischer Konflikte nur die Dynamik der Entfremdung der miteinander im Konflikt liegenden Personen - und wird somit selbst zum Motor des Prozesses, der in der Fachdiskussion als „Othering“ bezeichnet wird.

Es wundert nicht, wenn angesichts solch eines Deutungsmusters interkultureller moralischer Konflikte der moralische Anspruch auf universale Geltung moralischer Prinzipien mit sehr drastischen Worten als eine Form des „moralischen Genozids“ kritisiert wird, weil er letztlich nur eine Verallgemeinerung lokal begrenzter moralischer Werte sei (Chattopadhyay und de Vries 2013). Diese deutliche Kritik macht darauf aufmerksam, dass, anstatt auf diesen sehr scharfen Vorwurf wiederum mit der normativen Behauptung der Geltung universaler normativer Prinzipien zu antworten (z.B. Kovács 2010; Bracanovic 2011, 2013), ein besseres empirisch wie auch normativ reflektiertes Verständnis für die Relevanz kultureller Differenzen in moralischen Konflikten zu entwickeln ist - eine Perspektive, der sich auch die Herausgeber dieses Themenheftes anschließen.

Die ethische Reflexion interkultureller ethischer Konflikte steht schon durch ihre Themensetzung immer in der Gefahr, kulturelle Stereotype zu forcieren, wenn sie nicht deutlich unterscheidet zwischen dem hermeneutisch und dem empirisch zu rekonstruierenden Phänomen unterschiedlicher kultureller Prägungen moralischer Wertvorstellungen und der Behauptung, dass derartige kulturelle Differenzen eine normative Relevanz bei der Entstehung und Bearbeitung kultureller Konflikte haben. Am Beispiel verdeutlicht: So wenig man wird bestreiten können, dass wir als Herausgeber durch unsere jeweilige nationale und auch religiöse Herkunft kulturell unterschiedlich geprägt sind, so wenig kann man aus unserer religiösen und nationalen kulturellen Prägung Schlussfolgerungen darauf ziehen, was für jeden von uns in einem konkreten medizinethischen Konflikt relevante moralische Werte und Normen sind. Dies aber macht deutlich, dass ein näheres Verständnis der Relevanz kultureller Differenzen für ethische Konflikte im Gesundheitswesen sowohl eines besseren empirischen Verständnisses kultureller Differenzen als auch der normativen Reflexion auf die Relevanz dieser Differenzen bedarf.

Vor diesem Hintergrund fragen die Beiträge dieses Themenheftes in unterschiedlichen normativen und empirischen Perspektiven danach, wie kulturelle Differenzen in sozialen Beziehungen im Gesundheitswesen konstruiert werden, auf welche Weise sie normatives Gewicht gewinnen und wie mit moralischen Wertzuschreibungen im Rahmen der Konstruktion von kulturellen Identitäten ethisch verantwortungsvoll umgegangen werden kann.

Interessenkonflikt M. Coors, T. Peters und I. Ilkilic geben an, dass kein Interessenkonflikt besteht. 


\section{Literatur}

Brakanovic T (2011) Respect for cultural diversity in bioethics. Empirical, conceptual and normative constraints. Med Health Care Philos 14:229-236

Brakanovic T (2013) Against culturally sensitive bioethics. Med Health Care Philos 16:647-652

Chattopadhyay S, de Vries R (2013) Respect for cultural diversity in bioethics is an ethical imperative. Med Health Care Philos 16:639-645

Knipper M (2014) Vorsicht Kultur! Ethnologische Perspektiven auf Medizin, Migration und ethnisch-kulturelle Vielfalt. In: Coors M, Grützmann T, Peters T (Hrsg) Interkulturalität und Ethik. Der Umgang mit Fremdheit in Medizin und Pflege. Edition Ruprecht, Göttingen, S 52-69

Kovács J (2010) The transformation of (bio)ethics expertise in a world of ethical pluralism. J Med Ethics $36: 767-770$

Peters T, Grützmann T, Bruchhausen W, Coors M, Jacobs F, Kaelin L, Knipper M, Kressing F, Neitzke G (2014) Grundsätze zum Umgang mit Interkulturalität in Einrichtungen des Gesundheitswesens. Positionspapier der Arbeitsgruppe Interkulturalität in der medizinischen Praxis in der Akademie für Ethik in der Medizin. Ethik Med 26(1):65-75

Wimmer A (1996) Kultur. Zur Reformulierung eines sozialanthropologischen Grundbegriffs. Kölner Z Soz Sozpsychol 48:401-425 